\title{
Ultrafast electron-phonon decoupling in graphite
}

\author{
Kunie Ishioka, ${ }^{*}$ Muneaki Hase, ${ }^{\dagger}$ and Masahiro Kitajima \\ Advanced Nano-Characterization Center, National Institute for Materials Science, Tsukuba 305-0047, Japan \\ Ludger Wirtz \\ Institute for Electronics, Microelectronics, and Nanotechnology, CNRS, 59652 Villeneuve d'Ascq Cedex, France
}

Angel Rubio

European Theoretical Spectroscopy Facility, Universidad del País Vasco, Centro Mixto CSIC-UPV/EHU, Edificio Korta, Avenida de Tolosa 72, 20018 Donostia, Spain

and Donostia International Physics Center, P. Manuel de Lardizabal 4, 20018 San Sebastian, Spain

Hrvoje Petek

Department of Physics and Astronomy, University of Pittsburgh, Pittsburgh, Pennsylvania 15260, USA

and Donostia International Physics Center, P. Manuel de Lardizabal 4, 20018 San Sebastian, Spain

(Received 5 December 2007; revised manuscript received 14 January 2008; published 12 March 2008)

\begin{abstract}
We report the ultrafast dynamics of the $47.4 \mathrm{THz}$ coherent phonons of graphite interacting with a photoinduced nonequilibrium electron-hole plasma. Unlike conventional materials, upon photoexcitation the phonon frequency of graphite upshifts, and within a few picoseconds relaxes to the stationary value. Our first-principles density functional calculations demonstrate that the phonon stiffening stems from the light-induced decoupling of the nonadiabatic electron-phonon interaction by creating a nonequilibrium electron-hole plasma. Timeresolved vibrational spectroscopy provides a window on the ultrafast nonquilibrium electron dynamics.
\end{abstract}

DOI: 10.1103/PhysRevB.77.121402

PACS number(s): 78.47.-p, 63.20.-e, 81.05.Uw

Graphite possesses highly anisotropic crystal structure, with strong covalent bonding of atoms within and weak van der Waals bonding between the hexagonal symmetry graphene sheets. The layered lattice structure translates to a quasi-two-dimensional (2D) electronic structure, in which the electronic bands disperse linearly near the Fermi level $\left(E_{F}\right)$ and form pointlike Fermi surfaces. The discovery of massless relativistic behavior of quasiparticles at $E_{F}$ of graphene and graphite has aroused great interest in the nature of carrier transport in these materials. ${ }^{1-3}$ Because of the linear dispersion of the electronic bands in graphene, the quasiparticle mass associated with the charge carrier interaction with the periodic crystalline lattice nearly vanishes, leading to extremely high electron mobilities and unusual halfinteger quantum Hall effect. ${ }^{1,2}$ Since graphite has a quasi-2D band structure very similar to that of graphene, these electronic properties may be expressed also in graphite.

The electron-phonon $(e-p)$ interaction contributes to the carrier mass near $E_{F}$ and limits the high-field transport through the carrier scattering. The strong $e-p$ interaction in graphite is a distinctive characteristic of ineffective screening of the Coulomb interaction in semimetals. ${ }^{4,5}$ It is expressed in the phonon frequency shift by carrier doping 6 and the strong electronic renormalization of the phonon bands (Kohn anomalies). ${ }^{7}$ Time-resolved measurements on the optically generated nonthermal electron-hole $(e-h)$ plasma in graphite provide evidence for the carrier thermalization within $0.5 \mathrm{ps}$ both through electron-electron $(e-e)$ scattering and optical phonon emission. ${ }^{8}$ The nonthermal carriers decay nonuniformly in phase space because of the anisotropic band structure of graphite. ${ }^{5,9}$ Quasiparticle correlations in nonthermal plasmas can also be probed from the perspective of the co- herent optical phonons. In the present work, through the time-dependent complex self-energy (frequency and lifetime) of the $47 \mathrm{THz} E_{2 g 2}$ phonon of graphite, we study the transient changes in the $e-p$ coupling induced by the optical perturbation of the nonadiabatic Kohn anomaly.

To probe the ultrafast response of the coherent phonons, we perform transient anisotropic reflectivity measurements on a natural single crystal and highly oriented pyrolytic graphite (HOPG) samples. Because the phonon properties were identical, we report the results for HOPG only, whose better surface optical quality gave superior signal-to-noise ratio. The light source for the pump-probe reflectivity measurements is a Ti:sapphire femtosecond laser oscillator with $<10$ fs pulse duration. The fundamental output is frequency doubled in a $\beta$-barium borate crystal to obtain $395 \mathrm{~nm}$ excitation light. The $3.14 \mathrm{eV}$ photons excite vertical transitions from the valence $(\pi)$ to the conduction $\left(\pi^{*}\right)$ bands near the $K$ point. Pump power is varied between 5 and $50 \mathrm{~mW}$ (pulse fluence of $0.1-1 \mathrm{~mJ} / \mathrm{cm})$, while probe power is kept at $2 \mathrm{~mW}$. Details of the pump-probe measurements are described elsewhere. ${ }^{10,11}$ The anisotropic reflectivity change $\left(\Delta R_{e o}=\Delta R_{s}-\Delta R_{p}\right)$ eliminates the mostly isotropic electronic response to isolate the much weaker anisotropic contribution, which is dominated by the coherent phonon response. ${ }^{10}$

Figure 1(a) shows the anisotropic reflectivity change of graphite, $\Delta R_{e o} / R$, normalized to the reflectivity without pump pulse. After a fast and intense electronic response at $t=0$, the reflectivity is modulated at two disparate periods of 21 and $770 \mathrm{fs}$. The slower coherent oscillation was previously assigned to the Raman active interlayer shear phonon $\left(E_{2 g 1}\right.$ mode). ${ }^{12}$ The faster oscillation of $47.4 \mathrm{THz}$ or $1580 \mathrm{~cm}^{-1}$ is the in-plane $E_{2 g 2}$ carbon stretching mode 6 corresponding to the $G$ peak in the Raman spectra of gra- 

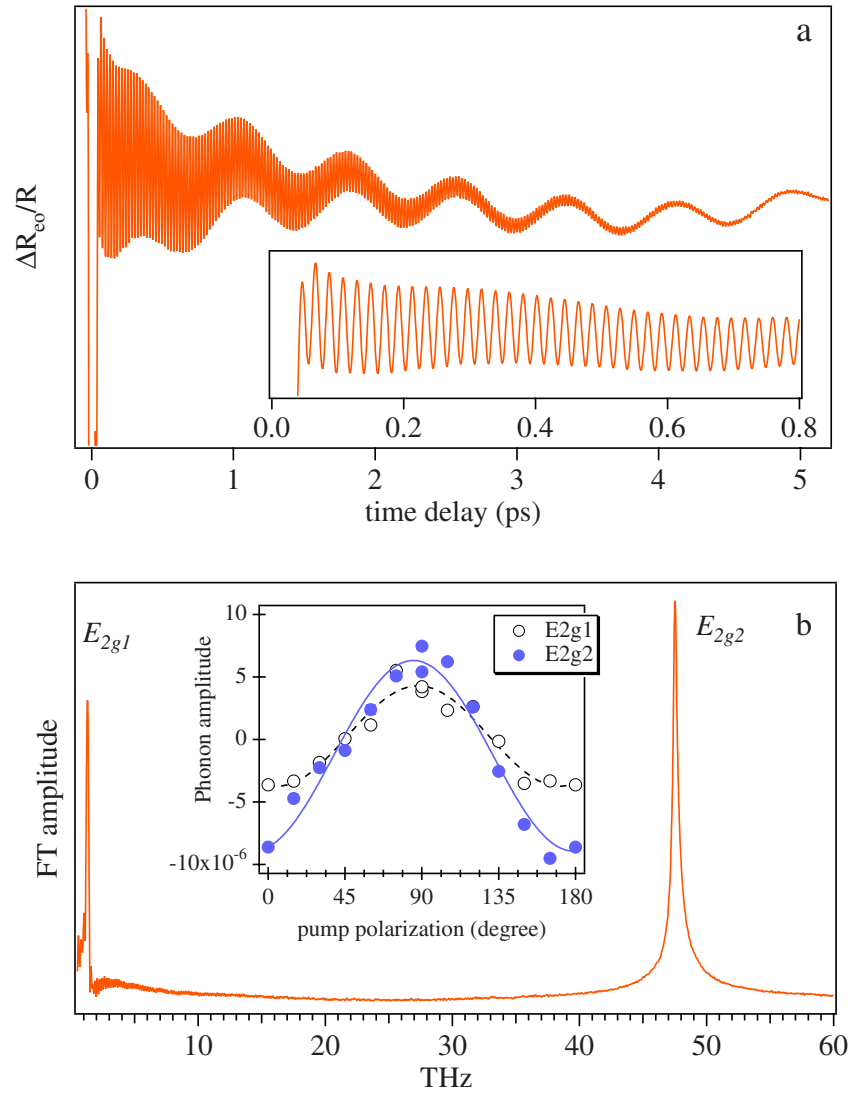

FIG. 1. (Color online) (a) Anisotropic reflectivity change $\Delta R_{e o} / R$ at pump power of $50 \mathrm{~mW}$. The inset shows an enlargement of the trace to show the high-frequency modulation. (b) FT spectrum of the time-domain trace in (a). Inset shows the pump polarization dependence of the amplitudes of the two coherent phonons $\left(A_{1}\right.$ and $\left.A_{2}\right)$ obtained from isotropic reflectivity $(\Delta R / R)$ measurement. The polarization angle is measured from the plane of incidence. The probe beam is polarized at $90^{\circ}$. Solid and broken curves are fits to $\cos 2 \theta$ function.

phitic materials. After decay of the electronic response, the reflectivity signal can be fitted approximately to a sum of damped oscillations: $f(t)=A_{1} \exp \left(-\Gamma_{1} t\right) \sin \left(2 \pi \omega_{1} t+\delta_{1}\right)$ $+A_{2} \exp \left(-\Gamma_{2} t\right) \sin \left(2 \pi \omega_{2} t+\delta_{2}\right)$. The amplitudes of both phonons, $A_{1}$ and $A_{2}$, exhibit a $\cos 2 \theta$ dependence on the pump polarization angle $\theta$ with respect to the optical plane, as shown in the inset of Fig. 1(b), confirming their generation through the Raman mechanism. ${ }^{12}$ Hereafter, we focus on the previously unobserved dynamics of the fast $E_{2 g 2}$ phonon.

We measure the laser fluence dependence of the amplitude $A_{2}$, dephasing rate $\Gamma_{2}$, and frequency $\omega_{2}$ of the $E_{2 g 2}$ phonon that are extracted from the fit of $\Delta R_{e o} / R$ to the damped oscillator model. The amplitude increases linearly with the fluence as expected for a $\pi-\pi^{*}$ transition with a single photon. As shown in Fig. 2, the dephasing rate decreases as the laser fluence is increased, which is contrary to the coherent phonon response observed for other materials. ${ }^{13-15}$ The frequency upshift at higher fluence in Fig. 2 is equally exceptional. Laser heating can be excluded as the origin because the $E_{2 g 2}$ frequency downshifts with temperature. ${ }^{16}$ In fact, the frequency upshift under intense optical excitation has not been observed experimentally or

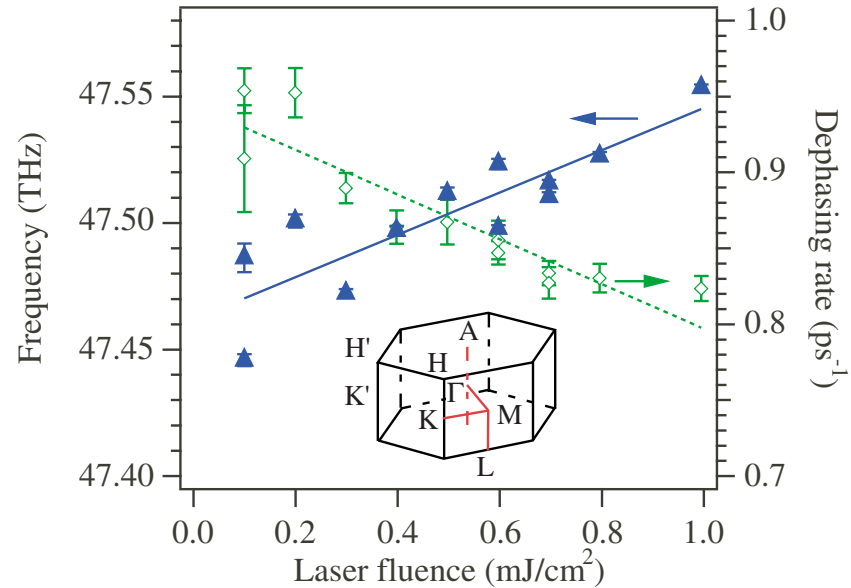

FIG. 2. (Color online) Laser fluence dependence of the dephasing rate $\Gamma_{2}$ and the frequency $\omega_{2}$ of the coherent $E_{2 g 2}$ phonon obtained from a fit to an exponentially damped oscillator function. The lines are to guide the eye. Inset shows the Brillouin zone of graphite.

predicted theoretically for graphite or any other solid.

To further characterize the unexpected frequency upshift, in Fig. 3, we analyze the transient reflectivity response with a time-windowed Fourier transform (FT). This analysis reveals that the phonon frequency blueshift occurs promptly (for delays of $<100 \mathrm{fs}$ its dynamics are obscured by the strong electronic response) and recovers to its nearequilibrium value after several picoseconds. With increasing laser fluence, the initial blueshift increases, while the asymptotic value converges on the $47.4 \mathrm{THz}$ Raman frequency. The experimental phonon frequency for $t>100 \mathrm{fs}$ follows a biexponential recovery, $\omega(t)-\omega(t=\infty)=\Delta \omega_{1} \exp ($ $\left.-t / \tau_{1}\right)+\Delta \omega_{2} \exp \left(-t / \tau_{2}\right)$, with time constants of $\tau_{1}=210 \mathrm{fs}$ and $\tau_{2}=2.1 \mathrm{ps}$, independent of excitation density. The time scales for the recovery are in reasonable agreement with the analysis of transient terahertz spectroscopy, which gave 0.4

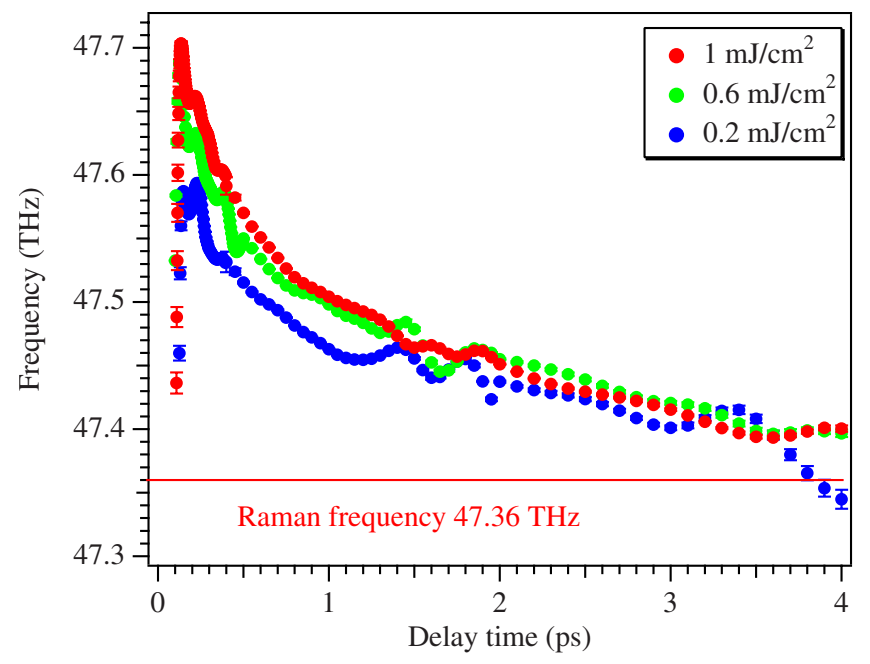

FIG. 3. (Color online) Time evolution of the $E_{2 g 2}$ phonon frequency, obtained from time-windowed FT, for different laser fluences. The widths of the Gaussian time windows are $80 \mathrm{fs}$ for $t$ $<0.4 \mathrm{ps}, 300$ fs for $0.4<t<2 \mathrm{ps}$, and 800 fs for $t \geq 2 \mathrm{ps}$. 
and $4 \mathrm{ps}$, respectively, for the carrier thermalization and carrier-lattice equilibration. ${ }^{8}$ The time evolution of the $E_{2 g 2}$ frequency implicates the interaction of coherent phonons with the photoexcited nonequilibrium carriers, as will be discussed below.

It is only recently that the observed anomalous dispersion of the high-energy phonon branches of graphite ${ }^{17}$ could be explained theoretically by a momentum dependent $e-p$ interaction (a Kohn anomaly), which leads to the renormalization (softening) of the phonon frequency. ${ }^{7}$ The standard use of the adiabatic approximation in the previous study, however, predicted that perturbing the electronic system by electron doping would result in a downshift of phonons at the $\Gamma$ point. Recent experiments and theoretical calculations have shown this approach to be inappropriate as the "nonadiabatic" electronic effects, where electrons near $E_{F}$ cannot respond instantaneously to the lattice distortion, become important for low dimensional materials such as graphene and nanotubes. ${ }^{18-20}$

We perform density-functional theory (DFT) calculations for a single sheet of photoexcited graphite with a computational method that accounts for the nonadiabatic effects. We use DFT in the local-density approximation as implemented in the code ABINIT. ${ }^{21}$ Core electrons are described by Trouiller-Martins pseudopotentials and the wave functions are expanded in plane waves with energy cutoff at 35 hartree. Phonons are calculated with density-functional perturbation theory. ${ }^{22}$ In order to ensure convergence of the $E_{2 g 2}$ phonon mode to within $0.01 \mathrm{THz}$, we use a large 61 $\times 61$ two-dimensional $k$-point sampling. ${ }^{23}$ Nonadiabatic effects are accounted for by keeping the electronic population fixed when computing the dynamical matrix. We neglect the effects of lattice relaxation on the phonon frequency since we checked that the effect of neutral excitation on the bondlength is very weak $(<0.001 \AA)$ for the appropriate excitation densities. Our approach is similar to the time-dependent perturbation scheme ${ }^{18-20}$ for the inclusion of nonadiabaticity in the combined treatment of phonons and electrons in graphite. Furthermore, it enables us to calculate the effect of an arbitrary electron occupation far from equilibrium such as created by the vertical excitation of $e-h$ pairs with $3.1 \mathrm{eV}$ photons. Our calculations demonstrate that nonadiabatic effects are important not only for charged graphite ${ }^{18-20}$ but also for graphite with neutral electronic excitation.

Because the photoexcited electron distribution is time dependent and, in principle, not known exactly, we employ three different limiting electronic distributions in our phonon calculation. "As-excited" distribution (AED), corresponding to the vertical excitation of $e$ - $h$ pairs with $3.1 \mathrm{eV}$ photons within an energy window of $\pm 0.2 \mathrm{eV}$, simulates the distribution right after excitation with a laser pulse having a finite spectral width. The laser fluence determines the amount of charge transferred from $\pi$ to $\pi^{*}$ bands. Nonthermal distribution (NTD), in which electrons are completely depopulated in an small energy window from top of the valence band to the bottom of conduction band, mimics the situation after the ultrafast ( $<100 \mathrm{fs}$ ) relaxation of the $e-h$ pairs toward $E_{F}$ by $e-e$ (Ref. 9) and $e-p$ (Ref. 24) scatterings. The width of the energy window is determined by the excited charge density. Such a distribution has been predicted in a recent micro-

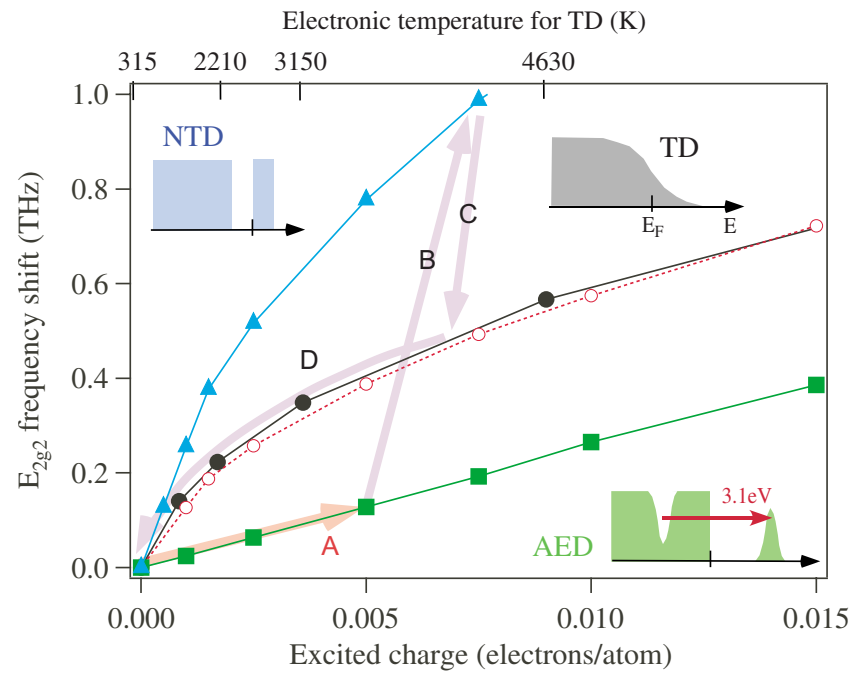

FIG. 4. (Color online) Calculated $E_{2 g 2}$ frequency change as a function of the excitation charge density for the AED (square), NTD (triangle), TD (filled circle), and ID (open circle). The top axis shows the corresponding electronic temperature $T_{e}$ for TD. Arrows show schematically the excitation and relaxation pathways for the $e$ - $h$ distribution: (A) quasi-instantaneous excitation by a laser pulse, (B) deexcitation within $<100$ fs through the creation of secondary $e-h$ pairs around the Fermi level $E_{F}$, (C) thermalization of the $e-h$ plasma in $\sim 0.2 \mathrm{ps}$, and (D) cooling down of the $e-h$ plasma in $\sim 2$ ps through optical phonon emission. Ultrafast phonon stiffening is ascribed to steps A and B. The highest density excitation (fluence of $1 \mathrm{~mJ} / \mathrm{cm}^{2}$ ) in our experiment corresponds to 5.8 $\times 10^{20}$ electron-hole pairs $/ \mathrm{cm}^{3}$ or 0.005 electrons/atom.

scopic calculation taking into account $e-p$ scattering. ${ }^{24} \mathrm{Be}-$ cause of a hot phonon distribution and a phase-space bottleneck near the $K$ points, a Fermi-Dirac distribution with a high electronic temperature is achieved at much later time [0.5 ps (Refs. 8 and 24)]. Hot thermal distribution (TD) simulates this situation after thermalization of the electronic system. To compare with the effect of static doping reported previously, ${ }^{18-20}$ we also present calculations with an ionized distribution (ID), in which electrons are removed from the top of the $\pi$ band.

Figure 4 shows that all the three excited state distributions, as well as the statically doped one, lead to a stiffening of the $E_{2 g 2}$ phonon. For a fixed density of the excited charge, the closer the $e$ - $h$ pairs are to the $E_{F}$, the more pronounced is their nonadiabatic interaction with the lattice, and therefore, the stronger is their effect on the phonon stiffening. We note that the stiffening is not accompanied by lattice deformation for the three excited distributions, contrary to the case of ID, for which the lattice both stiffens and contracts. The lattice stiffening for ID can be attributed to the depopulation of $\pi$ orbitals around the $K$ and $H$ points, which (i) suppresses the nonadiabaticity in the $e-p$ coupling and (ii) removes electrons with strong antibonding admixture. ${ }^{19,18}$ Because effect (ii) should also lead to a lattice contraction, the $\mathrm{C}-\mathrm{C}$ bond stiffening under the three excited distributions is attributed to effect (i). This implies that transfer of cold electrons and holes from near the $E_{F}$ to a hot population causes the stiffening along with the inability of the electronic system to 
follow the ions adiabatically. In contrast to the static doping studies, ${ }^{19,18}$ our observations on a neutral but nonequilibrium system address a phonon frequency shift solely of the electronic origin.

The strong dependence of the phonon stiffening on the $e-h$ distribution in Fig. 4 justifies the interpretation of the experimental ultrafast phonon frequency changes in terms of the temporal evolution of the photoexcited $e-h$ plasma. Creation of a highly nonthermal electronic population near the $K$ point (arrow A in Fig. 4) weakens the nonadiabatic $e-p$ coupling at $t=0$. Within a few tens of femtoseconds, the very efficient $e-e$ (Ref. 8) and $e-p$ (Ref. 24) scattering first bring the nascent $e-h$ distribution toward the $K$ point (arrow B), but still in a nonthermal distribution. The electronic thermalization (arrow C) is realized in about 0.2 ps. This hot-thermal distribution equilibrates with the lattice through optical phonon emission (arrow D) on 2 ps time scale.

In summary, we have explored the influence of the nonequilibrium $e$ - $h$ plasma on the femtosecond dynamics of the in-plane $E_{2 g 2}$ coherent phonon of graphite. The timedependent phonon frequency probes sensitively the time evolution of the transient electronic occupation distributions. The unusual electronic stiffening of the phonon can be attrib- uted to the excitation-induced reduction of the $e-p$ coupling due to quasi-2D electronic structure. The reduced real (frequency) and imaginary (decay rate) parts of the complex self-energy of the $e-p$ interaction increase the frequency and reduce the dephasing rate of the $E_{2 g 2}$ mode. Our results offer a paradigm of $e-p$ coupling, where nonequilibrium electrons impart exceptional properties to the lattice. Similar interactions are likely to govern the $e-p$ coupling in related graphitic materials, such as carbon nanotubes and graphene, which are of topical interest for high-performance, nanometer scale carbon-based electronic devices.

\section{ACKNOWLEDGMENTS}

The authors thank O.V. Misochko for supplying single crystal graphite. Calculations were performed at IDRIS (project 071827), Barcelona Supercomputing Center and UPV/EHU (SGIker Arina). This work is supported by Kakenhi 18340093, the EU Network of Excellence Nanoquanta (NMP4-CT-2004-500198), Spanish MEC (FIS2007-65702C02-01), French ANR (PJC05-46741), SANES project (NMP4-CT-2006-017310), NSF CHE-0650756, and Ikerbasque. *ishioka.kunie@nims.go.jp

†resent address: Institute of Applied Physics, University of Tsukuba.

${ }^{1}$ K. S. Novoselov, A. K. Geim, S. V. Morozov, D. Jiang, M. I. Katsnelson, I. V. Grigorieva, S. V. Dubonos, and A. A. Firsov, Nature (London) 438, 197 (2005).

${ }^{2}$ A. K. Geim and K. S. Novoselov, Nat. Mater. 6, 183 (2007).

${ }^{3}$ T. Ohta, A. Bostwick, T. Seyller, K. Horn, and E. Rotenberg, Science 313, 951 (2006).

${ }^{4}$ D. P. DiVincenzo and E. J. Mele, Phys. Rev. B 29, 1685 (1984).

${ }^{5}$ C. D. Spataru, M. A. Cazalilla, A. Rubio, L. X. Benedict, P. M. Echenique, and S. G. Louie, Phys. Rev. Lett. 87, 246405 (2001).

${ }^{6}$ M. S. Dresselhaus and G. Dresselhaus, in Light Scattering in Solids III, Topics in Applied Physics Vol. 51, edited by M. Cardona and G. Güntherodt (Springer, Berlin, 1982), Chap. 2.

${ }^{7}$ S. Piscanec, M. Lazzeri, F. Mauri, A. C. Ferrari, and J. Robertson, Phys. Rev. Lett. 93, 185503 (2004).

${ }^{8}$ T. Kampfrath, L. Perfetti, F. Schapper, C. Frischkorn, and M. Wolf, Phys. Rev. Lett. 95, 187403 (2005).

${ }^{9}$ G. Moos, C. Gahl, R. Fasel, M. Wolf, and T. Hertel, Phys. Rev. Lett. 87, 267402 (2001).

${ }^{10}$ M. Hase, M. Kitajima, A. M. Constantinescu, and H. Petek, Nature (London) 426, 51 (2003).

${ }^{11}$ K. Ishioka, M. Hase, M. Kitajima, and H. Petek, Appl. Phys. Lett. 89, 231916 (2006).

${ }^{12}$ T. Mishina, K. Nitta, and Y. Masumoto, Phys. Rev. B 62, 2908 (2000)
${ }^{13}$ M. Hase, M. Kitajima, S. I. Nakashima, and K. Mizoguchi, Phys. Rev. Lett. 88, 067401 (2002).

${ }^{14}$ E. D. Murray, D. M. Fritz, J. K. Wahlstrand, S. Fahy, and D. A. Reis, Phys. Rev. B 72, 060301(R) (2005).

${ }^{15}$ E. S. Zijlstra, L. L. Tatarinova, and M. E. Garcia, Phys. Rev. B 74, 220301(R) (2006).

${ }^{16}$ P. H. Tan, Y. M. Deng, and Q. Zhao, Phys. Rev. B 58, 5435 (1998).

${ }^{17}$ J. Maultzsch, S. Reich, C. Thomsen, H. Requardt, and P. Ordejón, Phys. Rev. Lett. 92, 075501 (2004).

${ }^{18}$ S. Pisana, M. Lazzeri, C. Casiraghi, K. S. Novoselov, A. K. Geim, A. Ferrari, and F. Mauri, Nat. Mater. 6, 198 (2007).

${ }^{19}$ M. Lazzeri and F. Mauri, Phys. Rev. Lett. 97, 266407 (2006).

${ }^{20}$ S. Piscanec, M. Lazzeri, J. Robertson, A. C. Ferrari, and F. Mauri, Phys. Rev. B 75, 035427 (2007).

${ }^{21}$ X. Gonze, J.-M. Beuken, R. Caracas, F. Detraux, M. Fuchs, G.-M. Rignanese, L. Sindic, M. Verstraete, G. Zerah, F. Jollet et al., Comput. Mater. Sci. 25, 478 (2002).

${ }^{22}$ S. Baroni, S. de Gironcoli, A. D. Corso, and P. Giannozzi, Rev. Mod. Phys. 73, 515 (2001).

${ }^{23} \mathrm{We}$ obtain an absolute value of $47.7 \mathrm{THz}$ for the $E_{2 g 2}$ mode, which is within $1 \%$ of the experimental frequency. Note that in the following, we calculate a shift in the phonon frequency, which is described much more accurately than the absolute value of the frequency.

${ }^{24}$ S. Butscher, F. Milde, M. Hirtschulz, E. Malic, and A. Knorr, Appl. Phys. Lett. 91, 203103 (2007) 